laws governing these details seems likely, not merely to provide an interesting chapter in chemical rate theory, but also to give firmer grounds for a claim that chemical reactive scattering is a subject worthy of the kind of attention which theoretical physicists have hitherto reserved for atomic and nuclear phenomena.

\section{antusas Chemistry and Biology}

from a Correspondent

LAST week (October 26 to 28) the Swedish Academy of Pharmaceutical Sciences sponsored a symposium on the chemistry and biological activity of cannabis which was held in the Foresta Hotel on the island of Lidingö, near Stockholm. The wealth of detail presented in the thirty-five contributions left no doubt that very significant advances in knowledge have been made since a somewhat similar conference was held two years ago (The Botany and Chemistry of Cannabis, edit. by C. R. B. Joyce and S. H. Curry; Churchill, London, 1970).

Work on the metabolism of the active tetrahydrocannabinol (THC) is now well advanced. Shortly after administration this highly insoluble substance is metabolized to the more soluble $7-\mathrm{OH}$, 6,7 dihydroxy and even more soluble acidic derivatives and several routes to the synthesis of these compounds and their radioactive labelling are now known (R. Mechoulam; J. L. G. Nilsson; T. Petrizilka). The THC seems to be fixed to some of the plasma lipoproteins (H. A. Klausner; $\mathbf{M}$. Widman) and to be rapidly transferred to the liver, spleen and the cortex of the adrenals (J. I. Heikkilä; Ryrfeldt J. Schou). Microsomal oxidation produces the more polar metabolites and they, and unchanged THC, are found in brain tissue shortly after administration (S. Agurell; S. Burstein; E. W. Gill). Correlation of concentrations in the brain with behavioural effects in small animals, primates and man has been established (M. E. Wall ; M. Willinsky ; W. M. McIsaac; E. A. Carlini; J. Hrbek) although it is still not certain whether THC or its metabolites are the active substance (W. D. M. Paton, who also pointed out the interesting connexion between fat soluble anaesthetics and the fat soluble THC).

A second impression was of the scientific complexity of the cannabis problem. The plant itself is a very plastic species with a large variation in the amounts of THC produced (J. W. Fairbairn). Apart from THC, another thirty related cannabinoids have been characterized and some of these may be active (F. W. H. M. Merkus ; T. B. Vree) or may modify the total activity; for instance, cannabidiol (CBD) prolongs phenobarbitone sleeping time in mice (W. D. M. Paton). But P. Waser's suggestion that CBD is converted to THC on smoking was not generally accepted. Reports on the amount of THC surviving the smoking process gave results varying from 20 to 80 per cent, so that it was difficult to know whether a $5 \mathrm{mg}$, $10 \mathrm{mg}$ or $20 \mathrm{mg}$ THC content of a reefer would produce a "mind-rocking experience" or not. Even the nomenclature is confusing; sixteen contributions used the benzpyran system of numbering and thirteen contributions the terpinoid, so that one had continually to remember that $\Delta^{1}$ and $\Delta^{9}$, or $7-\mathrm{OH}$ and $11-\mathrm{OH}$, were the same compounds. Steps are being taken to rectify this situation.

O. J. Braenden (Division of Narcotic Drugs, UN, Geneva) and M. C. Braude (National Institute of Mental Health, Rockville) described their ambitious programmes to coordinate effort and reduce overlap and confusion as much as possible.

A third important topic discussed by a panel, with the press present, was whether cannabis is harmful to health. Although definitive answers are not yet possible, it was interesting to note that, in the light of recent knowledge, nearly all the experts gave the impression they were against liberalization of use. The serious possibilities were raised of teratogenicity, tachycardia (especially if main-lining ever started), brain damage, accumulation in body fat, interference with microsomal enzymes (and therefore unknown effects on other drugs being taken for medicinal purposes), and carcinogenicity because deep inhalation is necessary for the full effect of a reefer. More general effects are a motivation leading to lack of drive and "dropping out", although N. E. Zinberg stated he had not noticed this markedly after a study of marihuana use in Vietnam. Unpleasant psychotic effects are also frequently reported and experimental work shows a slowing down of reaction time and inability to estimate time accurately; these effects can be serious when driving a car. Sir Harry Greenfield (International Narcotics Control Board) also pointed out that countries with a long history of cannabis use (for example, India, Egypt) are the most insistent on strict legal control.

A final impression gained was the hope that useful therapeutic substances may yet emerge and R. K. Razdan's work on crystalline water soluble derivatives of THC with interesting pharmacological properties may point the way. Abstracts only of each contribution will be published in Acta Pharmaceutica Suecica. Addresses of the speakers mentioned in this report may be obtained from Nature office.

\title{
Regulating the Initiation of Translation
}

THE notion that initiation factors in Escherichia coli, by regulating the binding of ribosomes to specific "start translating" signals in messenger RNAs, regulate the expression of particular genes at the level of protein synthesis, receives further support in next Wednesday's Nature New Biology. Berissi, Graner and Revel, who have studied the effect of initiation factors $\mathrm{F} 2$ and $\mathrm{F} 3$ on the binding of $E$. coli ribosomes to the RNA of bacteriophage MS2, a close relative of $\mathrm{R} 17$, conclude that factor F3 selectively promotes the initiation of translation of the MS2 coat protein cistron.

The binding of ribosomes to MS2 RNA in its native configuration depends on the presence of two initiation factors, F2 and F3. If, however, the MS2 RNA is partially denatured by exposure to dilute formaldehyde, a procedure developed by Lodish, ribosomes will bind to the RNA in the absence of factor F3 although factor F2 is still required. This finding provided Berissi et al. with an opportunity to ask whether or not F3 is capable of promoting the specific attachment of ribosomes to one or more of the initiation signals on MS2 RNA. In essence all they had to do was compare the ratio of the number of sites on partially denatured MS2 RNA bound to and protected by ribosomes in the presence of F2 alone or F2 and F3.
The results of such experiments proved to be all that anybody could hope for. With factor F2 alone the ribosomes bind to the initiation sites of the coat protein, RNA replicase and maturation protein in the ratio of $1: 2: 1$ but when factor F3 is also present, although the total number of ribosomes bound does not alter significantly, the ratio is shifted to $1: 0.5: 0.14$. In other words factor $\mathrm{F} 3$ specifically promotes the recognition of and binding to the initiation signal at the beginning of the coat protein cistron at the expense of the other two cistrons; factor $\mathrm{F} 3$ promotes the expression of the coat protein cistron.

Berissi et al. have started to look for other F3 factors which might specifically promote the translation of other cistrons or sets of cistrons, for the idea that gene expression is regulated in part by a battery of different F3 factors is obviously most attractive. So far they have discovered one other F3 factor, called F3B4 to differentiate it from F3B2 which promotes the recognition of the MS2 coat protein cistron. F3B4 does not stimulate the attachment of ribosomes to MS2 RNA but it does stimulate translation of phage $\mathrm{T} 4$ messenger RNA; it will be no surprise if the list of specific F3 factors rapidly lengthens in the near future. 